\title{
Representaciones sociales de la agricultura ecológica en Andalucía
} Social representations of the organic agriculture in Andalusia (Spain)

\section{Carmen Lozano Cabedo}

Profesora Ayudante Doctor.Facultad de Ciencias Políticas y Sociología. Universidad Nacional de Educación a Distancia (UNED)

clozano@poli.uned.es

\section{NUEVAS RURALIDADES}

MONOGRÁFICO COORDINADO POR SHARON R. ROSEMAN, SANTIAGO PRADO Y XERARDO PEREIRO

\begin{abstract}
RESUMEN actividad en la zona y, por extensión, en Andalucía.



PALABRAS CLAVE

representaciones sociales | aspectos socio-culturales | agricultura ecológica | Andalucía KEYWORDS

social representations | socio-cultural aspects | organic agriculture | Andalusia the members of the sector and others in the local population.
\end{abstract}

En los últimos años se ha generalizado un amplio abanico de representaciones e imágenes en torno a la producción ecológica, por parte de múltiples y diferentes actores sociales, tanto en lo que refiere a su definición y ámbito de actuación, como a las repercusiones de esta actividad sobre el ámbito económico, social, ambiental, económico e institucional. Este trabajo constituye un análisis de los discursos y las percepciones sobre la agricultura y la ganadería ecológica que posee la población rural andaluza y, en concreto, los habitantes de la comarca de la Sierra de Segura (Jaén). Se trata de un estudio sustentado en una metodología cualitativa, que trata de entender el imaginario que tanto los integrantes del sector como la población local proyectan sobre este sistema productivo y en qué medida esta imagen influencia la viabilidad futura de la

This work analyses different social actors' representations and images connected with the definition and implications of organic agriculture, as well as with the economic, social, environmental, economic, and institutional impact of this activity. We analyse the discourses about, and perceptions of, organic farming of the rural population of Andalusia and, in particular, those communicated by the inhabitants of the region of the Sierra de Segura (Jaén). In order to understand social representations of this activity and the influence that these perceptions would have for the future viability of organic agriculture in the area and, by extension, in Andalusia, the study has been conducted using a qualitative methodology and has examined the images of both

\section{Introducción (1)}

En los últimos años se ha generalizado un amplio abanico de representaciones sociales en torno a la producción ecológica (2). La capacidad que presenta esta actividad para abarcar múltiples dimensiones y dar respuesta a las demandas de una gran variedad de actores ha propiciado que se convierta en un ámbito de convergencia de los nuevos discursos y prácticas sobre el medio ambiente y el consumo, y en uno de los ejes sobre los que se sustenta la reorientación de la Política Agraria Común (PAC) y del desarrollo rural.

Desde la PAC se ha prestado especial atención a este sistema productivo, dada la necesidad de proponer un nuevo pacto entre agricultura y sociedad. En un momento en que el modelo agrario industrializado y productivista se ve cuestionado, la producción ecológica es valorada como elemento fundamental del proceso de reorientación de las directrices productivistas, intensivas y sectoriales que han caracterizado a la PAC, hacia una agricultura territorial, implicada en la conservación del medio ambiente y el paisaje, cuyo objetivo es la obtención de productos de calidad. Desde la esfera del 
consumo, marcada por los sucesivos escándalos alimentarios y por la demanda de productos distintivos, con una calidad y seguridad garantizadas (Lozano y Aguilar 2010), los productos ecológicos son apreciados porque ofrecen al consumidor un anclaje territorial (Zimmerman 1998) y porque garantizan que en su producción no se han utilizado insumos de origen químico o sintético. Esta actividad se ha convertido también en uno de los ejes principales de actuación del nuevo paradigma de desarrollo rural por su potencialidad para dinamizar socio-económicamente los territorios, establecer novedosos vínculos entre espacios rurales y urbanos, entre productores y consumidores, así como entre innovación y tradición (Darnhofer 2005).

En la actualidad coexisten diversas definiciones de la producción ecológica, así como visiones heterogéneas acerca del papel que esta actividad debe ejercer en la transformación del rol asignado al agricultor en el nuevo modelo de ruralidad, así como en la modificación de las relaciones entre actividad agraria, sociedad rural, naturaleza, territorio y alimentación (Banks y Marsden 2001). Estas conceptualizaciones pueden agruparse en torno a dos enfoques:

Por un lado, encontramos la definición desarrollada por la Federación de Movimientos de Agricultura Ecológica (IFOAM) que considera que la agricultura ecológica, orgánica, biológica o cualquier otra palabra equivalente, constituye un sistema holístico que da lugar a ecosistemas sostenibles, alimentos seguros, buena nutrición, bienestar animal y justicia social (IFOAM 1989). Esta concepción de la producción ecológica se caracteriza por abarcar diferentes dimensiones, pues valora el papel de esta actividad en la obtención de agroecosistemas ecológicamente sanos y autosostenibles (Daly 1991), cada vez más necesarios ante el avance del cambio climático y el agravamiento de las crisis alimentarias. Aboga también por la pluriactividad y la diversificación de ingresos (Kinsella et al. 2000), la estrategia de reducción de insumos, la producción para la venta local, o el comercio de proximidad. Apunta hacia un modelo de desarrollo que sea socialmente justo y ecológicamente responsable, así como por promover que los agricultores obtengan mayores cuotas de autonomía frente al poder de las multinacionales y los grandes empresas agro-alimentarias (Ploeg et al. 2000).

El carácter que adopta la producción ecológica en esta definición conecta directamente, según Sevilla Guzmán (1991) y Toledo (1993), con los manejos, usos y percepciones que han caracterizado históricamente a las sociedades campesinas. Es decir, implica la revitalización de formas tradicionales de producción, mayoritariamente campesinas, que fueron barridas por el empuje de la modernización. Ello ha llevado a considerar la producción ecológica como una de las actividades en las que se manifiestan signos de recampesinización (Ploeg 2008) (3). Resulta paradójico que los mismos rasgos "marginales" y "atrasados" que han sido denostados y que se consideraba que debían ser superados en aras de la modernización agraria, sean los que ahora la dotan de un valor añadido que le permiten conectar con las nuevas demandas de las sociedades postindustriales (Lash y Urry 1987) y con las novedosas aspiraciones ciudadanas de determinados grupos de consumidores.

Por otro lado, encontramos la definición desarrollada por las administraciones públicas en sus diferentes normativas. El interés por fomentar una definición breve, que permitiera regular y controlar esta actividad y que fuera fácilmente aplicable, propició que las primeras directrices para la regulación de esta actividad en Europa (4) definieran la agricultura ecológica como un conjunto de técnicas agrarias que excluyen la utilización de productos químicos de síntesis. Esta concepción se caracteriza por proponer una definición de la producción ecológica en negativo, resaltando los aspectos o prácticas que elimina o reduce, y por no tener en cuenta cuestiones relativas a la fertilidad del suelo, la promoción de la biodiversidad, el tratamiento del agua o la utilización de energía (Castañón 2001). También se ha señalado que esta forma de entender la agricultura ecológica se limita a proponer la sustitución de insumos de origen químico y sintético por otros certificados como "ecológicos" que son más caros, lo que perpetúa la dependencia de los agricultores de las empresas agroquímicas que controlan este mercado (Guthman 1998). El éxito que ha adquirido esta conceptualización radica, según Guzmán et al. (2000), en la definición sintética y divulgativa que propone, así como en su adecuación a los requerimientos actuales del mercado al no cuestionar el enfoque atomista, racionalista y mecanicista del modelo agrario industrializado. 
En este panorama en el que coexisten, o más bien se enfrentan (Clunies-Ross 1990), diferentes visiones acerca de lo que es y significa la agricultura ecológica, el nivel discursivo adquiere una gran importancia ya que es en este plano en el que los actores explican y argumentan sus formas de actuar, sus deseos, expectativas y motivaciones (Rubio y Varas 1997). Este trabajo pretende contribuir a este debate a través del análisis de las representaciones sociales que los diferentes actores de una comarca andaluza poseen de la agricultura ecológica, así como del papel que ésta tiene y puede tener como herramienta para la dinamización socioeconómica de las zonas rurales.

A partir de estas visiones se tratará de comprender los significados y valores asociados socialmente a este tipo de prácticas, así como el papel que desempeñan estas representaciones como factores de impulso o bloqueo a la expansión de la agricultura ecológica. Para ello distinguiremos entre la percepción que tienen de la misma los integrantes del sector (los productores, transformadores y elaboradores de alimentos ecológicos) y los que no están vinculados a la producción ecológica. Es decir, el resto de actores del territorio (agricultores convencionales, gerentes de empresas agroalimentarias, técnicos, representantes de la administración, integrantes de asociaciones culturales, de mujeres, etc.), a los que denominaremos "población local" (5).

Este trabajo describe, en primer lugar, las características de la zona de estudio elegida así como la metodología implementada. En segundo lugar, analiza el origen y evolución de las experiencias desarrolladas en la zona en torno a la producción ecológica, así como las diferentes estrategias y prácticas implementadas por los agricultores. En tercer lugar, estudia la narrativa que los integrantes del sector poseen acerca de este sistema productivo a través del análisis de los problemas encontrados a lo largo del proceso y de las barreras que perciben a la extensión de la misma en el territorio. En cuarto lugar, describe las visiones diferenciales de la producción ecológica que coexisten en la misma zona, distinguiendo entre la percepción que poseen de la misma los integrantes del sector y la que tiene el resto de la población del territorio. En último lugar, reflexiona acerca de las potencialidades que el conocimiento de estas representaciones sociales puede tener para la superación de las barreras que presenta la agricultura ecológica en esta comarca y, de forma más general, en Andalucía.

\section{Zona de estudio y metodología}

Esta investigación se ha realizado en Andalucía y, más concretamente en la comarca de la Sierra de Segura (Jaén). La elección de este territorio se justifica porque Andalucía es una de las regiones europeas con una evolución más significativa en el ámbito de la producción ecológica, ya que fue una de las primeras en establecer una normativa propia y en dotarse con instrumentos específicos para su regulación y control, y porque, desde hace años, concentra la mitad de los productores y hectáreas certificados en España.

La comarca de la Sierra de Segura fue una de las zonas pioneras en Andalucía en la implementación de experiencias de olivicultura ecológica. La singular trayectoria que ha tenido la producción ecológica en este territorio proporciona un marco para analizar las dificultades y problemas que encontró el sector en sus inicios, ilustrar sobre los cambios operados en el mismo, e identificar algunas de las barreras socioculturas y simbólicas que ralentizan la expansión de esta actividad. La situación de gran parte de esta comarca en el Parque Natural de las Sierras de Cazorla, Segura y las Villas ofrece además la posibilidad de estudiar cómo perciben los actores del territorio la relación naturaleza-cultura en un ámbito sujeto a una fuerte intervención ambiental y el papel otorgado a la agricultura ecológica en este imaginario.

Como este trabajo analiza las representaciones sociales generadas en torno a la agricultura ecológica por diferentes actores sociales, la metodología aplicada es básicamente cualitativa. Durante el tiempo que se realizó trabajo de campo en la zona (entre 2004 y 2007), se aplicó la técnica de la observación participante y se realizaron entrevistas en profundidad con objeto de comprender los procesos desde dentro y conocer en la perspectiva que los actores poseen de los mismos (Hammersley y Atkinson 2005). 


\begin{tabular}{|c|c|l|}
\hline Fases & \multicolumn{1}{|c|}{ Perfil } & \multicolumn{1}{|c|}{ Actor es entrevistados } \\
\hline $1^{2}$ Fase & $\begin{array}{c}\text { Informantes-clave } \\
\text { (10 entrevistas) }\end{array}$ & $\begin{array}{l}\text { Agentes de desarrollo, representantes de la } \\
\text { administración, elaboradores ecológicos, productores } \\
\text { ecológicos representativos }\end{array}$ \\
\hline $2^{2}$ Fase & $\begin{array}{c}\text { productores } \\
\text { ecológicos } \\
(30 \text { entrevistas })\end{array}$ & $\begin{array}{l}\text { Ganaderos, avicultores, agricultores (seleccionados } \\
\text { según el tipo de cultivo y municipio). }\end{array}$ \\
\hline \multirow{2}{*}{$3^{2}$ Fase } & $\begin{array}{l}\text { Población local } \\
\text { (53 entrevistas) }\end{array}$ & $\begin{array}{l}\text { Actores económicos (agricultores convencionales, } \\
\text { representantes de empresas agro-alimentarias, de } \\
\text { turismo y de asociaciones de empresarios) } \\
\text { - Actores institucionales (alcaldes, agentes de } \\
\text { Consejo Regulador de la Denominación de Origen) } \\
\text { - Actores sociales (integrantes de asociaciones } \\
\text { culturales, de mujeres, de jóvenes) }\end{array}$ \\
\hline
\end{tabular}

Cuadro 1. Número de entrevistas y perfil de los actores entrevistados. Fuente: Elaboración propia

En una primera etapa se efectuaron entrevistas abiertas a informantes-clave, es decir, a personas que tuvieron un papel determinante en el proceso de implantación de la agricultura ecológica en el territorio y en su posterior evolución (cuadro 1). A través de estos informantes obtuvimos una visión altamente cualificada de la génesis de esta actividad en la zona y pudimos identificar a los principales actores que estuvieron implicados en el proceso. Posteriormente, se entrevistó a un grupo de 30 productores ecológicos de la comarca: agricultores, ganaderos y avicultores, a los que se aplicó un guión de entrevista más estructurado para conocer sus motivaciones y trayectoria, los manejos desarrollados, el grado de organización interna existente en el sector de la agricultura ecológica o los problemas y potencialidades de este sistema productivo en la zona.

A medida que se fue avanzando en la investigación y analizando la información obtenida, se percibió la necesidad de superar las "fronteras" de este grupo. Consideramos que para conocer, en su globalidad, las limitaciones y potencialidades del sector de la agricultura ecológica en este territorio era necesario conocer la imagen, o mejor dicho imágenes, que la "población local" tenía de esta actividad. Se realizaron 53 entrevistas a un amplio abanico de actores económicos, institucionales y sociales de la comarca para estudiar la percepción generalizada de la agricultura ecológica entre los habitantes del territorio, los valores asociadas a la misma y el papel que le asignan en los procesos de desarrollo territorial.

La observación participante ha tenido un papel fundamental en el desarrollo de esta investigación, dado que nos permitió analizar, de primera mano, el tipo de relación establecida entre los productores ecológicos, así como entre ellos y otros actores de la comarca, así como corroborar datos y contrastar las opiniones obtenidas en las entrevistas (Jociles 1999).

\section{Caracterización de la agricultura ecológica en la Sierra de Segura}

La Sierra de Segura está ubicada en el ámbito nororiental de la provincia de Jaén y presenta rasgos comunes a la mayoría de las áreas de montaña españolas: despoblación, envejecimiento y masculinización de su población. Las actividades económicas de la zona han estado condicionadas por la presencia de pendientes pronunciadas, clima extremo y suelos poco fértiles. Estas características propiciaron el desarrollo de un modelo caracterizado por la diversificación y la interrelación entre actividades. Una tendencia que ha variado en las últimas décadas pues la economía de la zona se 
sustenta, actualmente, en un único cultivo, el olivar, que ocupa el $78 \%$ de la superficie labrada de la comarca.

Al ser un olivar de montaña, con una productividad mucho más baja y unos costes más elevados que en otras zonas, los agricultores y agricultoras de esta comarca, junto con diversas instituciones, optaron desarrollar diversas estrategias para fomentar la calidad de sus aceites al considerar que era la única vía para competir con áreas más productivas. En 1979 se reconoció la Denominación de Origen del aceite "Sierra de Segura" (6) y a partir de 1985 comenzó a implementarse una iniciativa en torno al olivar ecológico. Estas experiencias coincidieron en el tiempo con la creación del Parque Natural de las Sierras de Cazorla, Segura y las Villas en 1986, en el que una parte sustancial del territorio quedaba integrado, lo que les llevó a pensar que la promoción del binomio "Parque Natural-Agricultura Ecológica" podría otorgar valor añadido al territorio y a los productos agroalimentarios obtenidos en el mismo.

Como ámbito de aplicación de la iniciativa de olivar ecológico se eligió un pequeño municipio: Génave, pues contaba con una superficie de olivar no demasiado extensa (1.268 has) y estaba ubicado en una de las esquinas del Parque Natural. A ello había que sumar que la población del municipio era reducida (837 habitantes) y que sus agricultores estaban integrados en una única cooperativa, lo que permitió involucrar a todo el pueblo en el proyecto (Pajarón 2001). En 1985 se inició la reconversión de los olivares hacia la producción ecológica en un proceso que estuvo plagado de dificultes por la ausencia de información y asesoramiento técnico, la escasez de productos autorizados para suplir la pérdida de aportes suplementarios y la caída de la productividad de los árboles y, sobre todo, por la dificultad de introducir en el mercado un aceite etiquetado como "biológico". Hay que tener en cuenta que cuando comenzó esta experiencia no existía reconocimiento oficial de esta actividad pues el primer reglamento español no se aprobó hasta 1989 y hay que esperar hasta 1991 para que se estableciera el Reglamento europeo y se creara en Andalucía un instrumento propio para la regulación del sector: el Comité Territorial Andaluz de Agricultura Ecológica.

La combinación de estos factores propició que, tras dos años de producción y sin haber conseguido sacar el producto al mercado, la cooperativa decidiera volver a la producción convencional. Esta decisión no fue unánime pues un grupo de 46 agricultores con unas 400 has de olivar decidió continuar con la experiencia y crearon su propia cooperativa: Sociedad Cooperativa Andaluza Sierra de Génave, integrada únicamente por productores ecológicos. Esta situación provocó una fractura social en el pueblo, que terminó dividido en dos grupos: "los convencionales" y "los ecológicos", o como ellos mismos se denominan: "Ios de arriba” y "los de abajo“, y generó multitud de conflictos que, todavía hoy, perviven (Lozano 2010).

A pesar de los problemas encontrados, los agricultores ecológicos de Génave continuaron su andadura y su ejemplo fue calando en la zona pues diferentes productores del territorio iniciaron la reconversión a la agricultura ecológica. En 2005, momento en que se inició el trabajo de campo en la zona, la Sierra de Segura contaba con 146 agricultores ecológicos y unas 1.800 hectáreas certificadas, de las cuales el $71 \%$ se dedicaba al olivar. La preponderancia de este cultivo podría indicar que estamos ante en un panorama social homogéneo, con unas expectativas, manejos y estrategias similares. Sin embargo, para entender las características que presenta el sector de la agricultura ecológica (7) en este territorio hay que realizar una distinción entre dos grupos: la sub-unidad Sierra de Génave y la sub-unidad Sierra de Segura (Ojeda 2003).

La sub-unidad Sierra de Génave se compone por los 84 socios de la cooperativa ecológica de Génave y por unas 1.000 has repartidas por los términos municipales de Génave y Villarrodrigo. Prácticamente la totalidad de esta superficie está dedicada al olivar, con lo que se mantiene la línea orientada al monocultivo del olivar tan extendida en la agricultura convencional de la comarca. Este grupo se caracteriza, a grandes rasgos, por haber realizado una práctica de sustitución de insumos considerando que, aunque los productos autorizados son más caros, facilitan bastante las labores e inciden positivamente en la productividad del olivo. Prefieren, asimismo, arar la tierra a mantener la cubierta vegetal alegando la mayor carga de trabajo que supone mantener la hierba, estar pendiente de la explotación para eliminarla en el momento en que ésta empiece a competir con el olivo por el agua, y 
realizar estas labores sobre terrenos en pendiente de los olivares:

"Con el terreno que tenemos no puedes meter una desbrozadora o un tractor, hay que hacerlo a mano y eso es mucho trabajo. Si el terreno fuera más apacible, pues sí, metes una desbrozadora o un tractor y lo quitas todo, pero a mano, madre mía" (agricultor ecológico, Sub-Unidad Sierra de Génave, 15/02/05).

Una de las características fundamentales de este grupo es que presenta una fuerte cohesión interna, lo que les ha permitido desarrollar estrategias comunes tanto a la hora de producir, como de comercializar y vender su producto. La cooperativa ha asumido las labores de molturación y elaboración del aceite y actúa como plataforma para la información y el asesoramiento de sus socios, así como para la adquisición de insumos a precios más bajos. Con los años ha ido ampliado su ámbito de actuación mediante la construcción de su propia planta embotelladora y adoptando estrategias de coordinación vertical, al integrarse en la cooperativa de $2^{\circ}$ Grado "Olivar de Segura" que, desde ese momento, se ha encargado de comercializar su producto. La especial trayectoria seguida por esta sub-unidad, los múltiples problemas encontrados a lo largo del camino, pero también los éxitos que han cosechado les ha otorgado un fuerte sentimiento de grupo, lo que se refleja en sus prácticas y discursos que tienden a ser relativamente homogéneos.

La sub-unidad Sierra de Segura está integrada por 62 productores que, desde mediados de los años 90, han ido realizando su conversión hacia la agricultura ecológica. Sus integrantes están repartidos en 10 municipios lo cual nos da una idea de la dispersión y la distancia física existente entre ellos. Aunque el cultivo del olivar sigue siendo predominante en este grupo, se puede encontrar una producción más diversificada que en el caso anterior: almendros, productos hortofrutícolas, ganadería, etc., estableciéndose, en algunos casos, asociaciones entre los mismos. Ambas circunstancias: diversidad y lejanía constituyen, según los informantes, un freno al establecimiento de estrategias comunes y a la creación de mecanismos de coordinación. La principal característica de este colectivo es que utilizan los insumos ecológicos autorizados en menor medida que la otra sub-unidad, en favor de la implementación de un conjunto de prácticas agroecológicas que, según los informantes, requieren más tiempo, dedicación y conocimientos que la técnica de sustitución de insumos, pero generan mayores beneficios para el medio ambiente.

Un ejemplo claro de esta orientación es que la mayoría de las explotaciones de olivar de este segundo grupo mantiene las cubiertas vegetales. Aunque admiten que su manejo es complicado y requiere grandes dosis de esfuerzo, creen que es un elemento básico en la gestión del agroecosistema ya que permite combatir la erosión tan acentuada en esta zona, mantener la fertilidad del suelo y promover la biodiversidad. El desarrollo de esta práctica les permite además acogerse a las ayudas destinadas a la lucha contra la erosión en el olivar y percibir unos ingresos suplementarios.

"Un agricultor para que se pueda considerar como tal tiene que cuidar lo que es la base de su sistema de producción, que es el suelo, no se pierda. Y los convencionales la están perdiendo. Pan para hoy, desierto para mañana. Claro, cuando tú dejas la hierba, la producción es menor porque compiten por el agua. Sin embargo, en el momento en que dejas la hierba, la erosión desaparece. Y eso para mí es una satisfacción" (agricultor ecológico, sub-unidad Sierra de Segura, 17/01/2005).

La escasa articulación interna entre los productores de esta sub-unidad y la ausencia de otras almazaras ecológicas ha propiciado que, durante años, los olivicultores de este grupo dependieran de la cooperativa de Génave para la molturación de la aceituna y para el envasado, comercialización y venta del aceite. El interés por elaborar un producto propio ha llevado a los agricultores de esta sub-unidad a experimentar diferentes alternativas (molturación en almazaras convencionales, creación de microalmazaras o de una cooperativa con dos líneas de molturación independientes, una para la aceituna ecológica y otra para la convencional), aunque la mayoría de estas experiencias ha tenido una trayectoria breve al no contar con un número suficiente de productores que la respaldase.

Más allá del ámbito del olivar, la comercialización es un problema todavía más acuciante para el resto de 
cultivos y actividades desarrolladas en la comarca, tal y como destacan Acuña y Ranocchiari (2012), lo que ha propiciado que muchos agricultores y ganaderos ecológicos, ante la ausencia de canales propios, tengan que vender su producto como si fuera convencional. En los últimos años, esta tendencia ha comenzado a variar y en 2008 encontrábamos 4 empresas dedicadas a la elaboración de conservas vegetales y pan ecológico, por lo que también se aprecia una mayor diversidad en este ámbito con respecto a la otra sub-unidad.

\section{Los problemas y retos a los que se enfrenta la producción ecológica en el territorio}

Para obtener una visión completa de las características que presenta la agricultura ecológica en la Sierra de Segura, en este epígrafe se analizan, a través de la "mirada" de los protagonistas, los problemas encontrados a lo largo de este proceso, así como los obstáculos que perciben para su desarrollo futuro.

\subsection{Los problemas de la agricultura ecológica en la zona}

Como ya hemos destacado, el principal problema que los agricultores ecológicos de la Sierra de Segura encontraron en el desarrollo de esta actividad fue el de la comercialización y venta de su producto. Los integrantes de la sub-unidad Sierra de Génave consideran que este fue el motivo de la escisión entre "ecológicos" y "convencionales" en la localidad y el obstáculo más importante que tuvo que salvar el grupo en sus primeros años. Los olivicultores de la sub-unidad Sierra de Segura indican que existen múltiples trabas para comercializar a través de canales propios, tanto internas como externas al territorio, y que resulta difícil introducir nuevas marcas de aceite ecológico diferentes a las que controla la Cooperativa Sierra de Génave. Esa situación se agudiza entre los agricultores que desarrollan otros cultivos, así como entre los ganaderos pues apenas existen vías para vender su producto como "ecológico".

Otro de los obstáculos que se menciona de forma recurrente es el de índole institucional y administrativo ya que casi todos los informantes han hecho referencia, a lo largo de su discurso, a la gran cantidad de trámites y "papeleo" que hay que realizar. Se quejan también de los inconvenientes que genera, en sus proyecciones económicas a corto y medio plazo, el continuo incumplimiento de las administraciones en el pago de las subvenciones. Consideran que esta demora en el cobro de las ayudas no solo les afecta a ellos, sino que también crea falsas expectativas respecto a la rentabilidad atribuida a la producción ecológica y desalienta a posibles productores que quieran iniciar la reconversión.

"Los mayores problemas son a la hora de cobrar las subvenciones de la agricultura ecológica que no llegan, que te embarcas, te metes en cosas y es que... no te llegan. A la fecha que estamos todavía no hemos cobrado las del año pasado" (agricultor ecológico, sub-unidad Sierra de Génave, 15/02/2005).

Un amplio número de agricultores resalta que no reciben suficiente apoyo por parte de las administraciones y que éstas tienen un doble rasero a la hora de tratar estos temas ya que, aunque a nivel discursivo les otorgan un fuerte respaldo porque "queda bien en la foto", en la práctica ponen multitud de trabas que obstaculizan el proceso. Algunos van más allá y señalan que el principal obstáculo para el desarrollo de la agricultura ecológica es el propio diseño de la Política Agraria Común que, al mismo tiempo que apoya la agricultura industrializada, destaca los beneficios de la agricultura ecológica.

"Nosotros tenemos que demostrar que lo estamos haciendo bien cuando está permitido envenenar todo" (agricultor ecológico, sub-unidad Sierra de Segura, 01/02/2005).

En lo relativo a las técnicas y manejos desarrolladas en agricultura ecológica, y en consonancia con otros estudios realizados en la región (García Trujillo 2001), la mayoría indica que los principales obstáculos en este ámbito han sido la falta de información y asesoramiento, un cierto "desamparo" a la hora de afrontar el período de reconversión, así como puntuales problemas de manejos o plagas. 
En el discurso de los entrevistados ha surgido recurrentemente, aunque no de forma tan explícita como cuando se habla de otro tipo de dificultades, el problema que supone la presión social. Muchos informantes hacen referencia a las continuas burlas y bromas de las que son objeto por el hecho de ser ecológicos, dado que esta actividad es considerada como una actividad marginal y "una cosa de locos". Esta práctica, que tienen como finalidad desprestigiar dicha actividad y por extensión a las personas que la practican (Morgan y Murdoch 2000), refleja un método de sanción social bastante usual en las sociedades campesinas tradicionales cuya finalidad era la de igualar a sus integrantes, evitando la "diferenciación" entre sus miembros y, por tanto, la desestabilización de la comunidad (Foster 1974).

"Parece que somos la oveja negra, estamos un poco discriminados. Como nos salimos de la regla de lo normal, de lo que se considera normal. La gente no hace más que meterse en nuestras olivas, para ver si no tienen, para reírse. Somos como la burla de aquí" (agricultora ecológica, sub-unidad Sierra de Segura, 29/09/2005).

"En el colegio le dicen a mis hijos: 'tu padre tiene el olivar abandonado, tu padre no sabe ser agricultor'. Los mismos niños..." (agricultor ecológico, sub-unidad Sierra de Segura, 06/09/2005).

\subsection{Principales barreras a la expansión de la agricultura ecológica en el territorio}

Para la mayoría de los integrantes del sector, el fomento de la producción ecológica constituye una de las estrategias más viables de desarrollo de la comarca no sólo porque promueve una producción de calidad, sino también porque es una actividad que impulsa la conservación del medio ambiente y se ajusta a la perfección a la imagen de "sostenibilidad" que se trata de incentivar desde el Parque Natural. Aunque esta opinión está bastante generalizada entre los agricultores ecológicos, cuando se les pregunta acerca de las posibilidades de que este sistema productivo se expanda por la zona se muestran mucho más pesimista ya que creen que existe múltiples factores que juegan en su contra.

Consideran que el obstáculo principal es la menor rentabilidad de esta actividad, especialmente por la menor cosecha obtenida. Se prioriza, ante todo, el volumen de producto obtenido, sin tener en cuenta otras cuestiones como la percepción de subvenciones complementarias o la obtención de un precio más elevado por el producto. Por tanto, podemos decir que uno de los principales frenos al desarrollo del sector estriba en la preponderancia que, aún hoy, tiene la lógica productivista en la zona. También se considera que un problema fundamental es la mayor carga de trabajo, tiempo y dedicación que esta actividad requiere y que muy pocas personas del territorio parecen dispuestos asumir (Lozano 2011).

El mantenimiento de la cubierta vegetal por parte de algunos agricultores ecológicos es, según los integrantes del sector, uno de los principales elementos disuasorios, dada la animadversión que se tiene hacia las cubiertas herbáceas en la zona. Una imagen negativa que viene asociada al desconocimiento generalizado de la población local respecto a esta actividad. Los productores entrevistados ponen de relieve que, a pesar de la multitud de cursos y jornadas que se han organizado en la comarca, no se ha sabido llegar a la gente y explicar de forma clara qué es la producción ecológica.

"Se ha saturado con pocos argumentos, a la gente hay que convencerla con algo y como tiene menos producción, la oliva se pone fea, pues no han convencido los argumentos a favor, que deberían haberse expuesto de forma más clara y no el 'esto es bueno porque sí'. Aunque vean que a los agricultores ecológicos les va bien, pero como ellos siguen teniendo más producción que la ecológica, pues les da miedo" (Agente de Desarrollo Local, 17/01/2005).

Otro de los factores disuasorios identificados es el escaso reconocimiento social de esta actividad y la presión social a la que se somete a sus integrantes pues es un elemento que desalienta a los posibles interesados en la reconversión. Así, algunos de los socios de la Cooperativa Sierra de Génave nos indicaban que, aunque les consta que algunos agricultores convencionales querrían "bajarse" a la cooperativa y hacerse ecológicos, no se atreven por las consecuencias que ello les podría acarrear a nivel social y económico.

La desarticulación interna del sector no suele ser identificada como un obstáculo al desarrollo de la 
actividad en el territorio, pero cuando se les interroga sobre dicha cuestión, la mayor parte reconoce que este elemento es una debilidad y consideran que sería necesaria una mayor cohesión entre ellos. Aunque dudan de que un proyecto así pudiera llevarse a cabo, admiten que el establecimiento de lazos entre ambas sub-unidades les permitiría afrontar los problemas que presenta la agricultura ecológica en la zona, asumir el reto de la comercialización y venta del aceite y fomentar una imagen consolidada y homogénea del "producto ecológico de la Sierra de Segura".

\section{Percepciones diferenciales de la agricultura ecológica en la Sierra de Segura}

A lo largo de este epígrafe realizaremos un acercamiento a las diferentes visiones de la agricultura ecológica presentes en el territorio. Se analizará la percepción que la "población local" y el sector ecológico tiene de la producción ecológica y de los atributos que la caracterizan. Asimismo, se describirán los discursos diferenciales que emergen entre los dos grupos de productores ecológicos identificados y que ponen en evidencia la coexistencia de diversas formas de concebir esta actividad incluso entre aquellos que la practican.

\subsection{Representaciones sociales de la agricultura ecológica entre la "población local"}

La percepción que los diferentes actores del territorio entrevistados tienen de esta actividad puede agruparse en torno a cuestiones: percepciones relacionadas con las técnicas propias de la agricultura ecológica, y percepciones vinculadas a los rasgos personales de los agricultores ecológicos o a la peculiar trayectoria del sector en la zona.

\subsubsection{Percepciones relacionadas con las técnicas propias de la agricultura ecológica}

La "población local" suele considerar a la agricultura ecológica como una actividad menos rentable que la convencional, pues no sólo la producción es menor, sino que los insumos son más caros. Unos condicionantes que no se ven compensados, a ojos de los entrevistados, por el hecho de que haya más subvenciones o de que sus productos se vendan a un precio superior. Muchos informantes aluden, como un factor claramente negativo de la producción ecológica, a que hay que estar más pendiente de la explotación y dedicarle más horas de trabajo. Lo que nos parece más relevante de este discurso es que se ajusta a las apreciaciones realizadas por los integrantes del sector cuando se les interrogaba acerca de las barreras que ralentizan el desarrollo de la producción ecológica en la zona. Un porcentaje importante de los actores entrevistados considera que la reconversión supone una vuelta atrás, un retroceso en el camino hacia la modernización, ya que supone la renuncia a muchos adelantos técnicos, tanto en forma de productos, como de maquinaria.

"Pero es que la producción que tiene un olivar ecológico es la mitad o menos que la convencional. Y el gasto es superior, encima. Porque yo hago cualquier cosilla en los olivos y me voy. Yo trabajo en la hostelería. Yo le hago cuatro cosillas, las podo, las cojo, las curo, le curo el suelo y ya está" (agricultor convencional, 30/10/2007).

También se suele vincular esta actividad con abandono o dejadez de los cultivos, percepción que parece sustentarse en dos argumentos. Por un lado, en la actuación de determinados agricultores que inscribieron olivares abandonados en agricultura ecológica para cobrar las subvenciones, pero a los que no impulsaba ningún otro tipo de motivación. Por otro, en el hecho de que, tras la reconversión, los cultivos experimenten una reducción de su productividad como consecuencia de la restricción de aportes y de la adaptación a los nuevos manejos. Los olivos, por ejemplo, suelen experimentar una merma en la cantidad de aceituna obtenida por árbol y adoptan un aspecto amarillento, lo que tiende a asociarse con fealdad, enfermedad y, por tanto, con un conjunto de connotaciones negativas.

"Yo pienso que la agricultura ecológica, si tú trabajas en ella. Si le echas estiércol, sí. Pero es que aquí los ecologistas que hay, eso es gandulismo. Aquí, ecológicas es no les hago nada. Si cae la breba y me da cosecha, pues... eso no..." (Gerente cooperativa aceite convencional, 02/03/2006). 


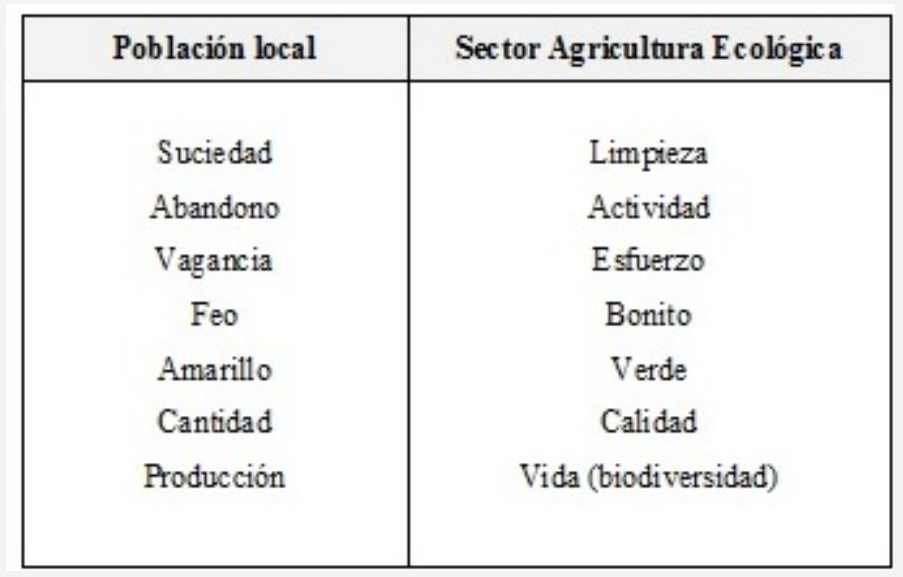

Cuadro 2. Discursos de los actores sobre la imagen de los olivares ecológicos.

Fuente: Elaboración propia

En los discursos de los diferentes actores del territorio aparece, recurrentemente, la alusión al aspecto de los cultivos, destacando si están verdes o amarillos, bonitos o feos, limpios o sucios. Tales categorías (cuadro 2) se utilizan de forma generalizada para alabar o denigrar determinadas fincas y, de forma extensiva, al agricultor que las gestiona, a los que se tacha de vagos, perezosos o dejados.

"Si las olivas no hay más que verlas. Tú vas a un olivar ecológico y están las olivas rubias, que no tienen de aquí, están envueltas en hierba..., si la hierba se come todo lo que pilla" (agricultor convencional, 02/03/2006).

El ejemplo más claro de esta perspectiva es la consideración de que el mantenimiento la cubierta herbácea en los olivares es sinónimo de "falta de limpieza o suciedad" en la finca. Como desde la lógica de estos actores, la fauna y flora auxiliar son consideradas perjudiciales, o en el mejor de los casos inútiles, ya que no poseen una función alimenticia, no se valoran las potencialidades de estos elementos en la promoción de la fertilidad, la prevención de enfermedades, etc. Es más, su presencia en la explotación es considerada como muestra de irracionalidad y como signo de desorden. Esta clasificación supone una clara muestra del arraigo que poseen los criterios racionalistas y mecanicistas que caracterizan al modelo industrializado de agricultura (Guzmán et al. 2000). Dicha percepción conduce a los agricultores convencionales a considerar que los manejos desarrollados en Génave pueden clasificarse como una forma "más razonable" de implementar la producción ecológica, ya que al de no dejar que la flora arvense "invada" la finca, la imagen de sus olivos es semejante a la de los convencionales, que se mantienen "limpios" durante todo el año.

"Ya te digo, yo en Génave he visto algunas que están muy bien. No tienen hierba. Hasta las de al lado no eran ecológicas y estaban peor" (agricultor convencional, 04/03/2006).

La agricultura ecológica se identifica también con un proyecto institucional, con el interés de la administración por implementar determinadas iniciativas que legitimen la "sostenibilidad" y "el respeto medioambiental" de su programa político. Según los actores locales, el interés por fomentar esta actividad en los Parques Naturales pone de relieve la escasa importancia que se otorga a la misma, ya que la confinan a zonas con una agricultura marginal, pero no a resolver los problemas de las áreas más contaminadas por la merma que ello supondría para su productividad (Santamaría 2009).

“El cuento de la agricultura ecológica es un fracaso rotundo de la administración... Eso es un cuento que sólo sirve para cumplir la cuota del programa $X$ del partido político $Z$, que dicen que tienen que cumplir... Como el protocolo de Kyoto, una cosa parecida. La cuota ecológica se pretende cumplir con los Parques Naturales y la agricultura ecológica... Yo creo que para respetar la ecología y el medio ambiente se tiene que hacer en todo a la vez. Todas las cosas a la vez. No una cosa aislada aquí" (Presidente cooperativa aceite convencional, 06/03/2006). 


\subsubsection{Percepciones vinculadas a las características personales de los agricultores ecológicos}

A los agricultores ecológicos se les conoce en la zona como "los hippies" o "los ecologistas". Con el término "hippies" se hace referencia a que son personas que practican un tipo de agricultura alternativa y marginal, que está fuera de los cauces de lo que se considera "normal" (8). Una denominación que tiene como objetivo banalizar esta actividad (Naredo 1983), así como otorgarle un carácter arcaico y desfasado, obstaculizando la percepción de las innovaciones en técnicas y en conocimientos que conlleva este sistema productivo (Morgan y Murdoch 2000). También se les atribuye el apelativo de "ecologistas", dando a entender con ello que anteponen los objetivos ambientales de su explotación a los económicos y que presentan una actitud extremista con respecto a este supuesto. Aunque, ciertamente, en la zona hay varios productores ecológicos que pertenecen al Colectivo Ecologista Segura Verde o comparten este ideario, también hay muchos que hacen hincapié en diferenciar que ellos son "ecológicos" y no "ecologistas" para desvincularse de esta imagen peyorativa y de las características que se le atribuyen. En otras palabras, el interés que la producción ecológica otorga al fomento de la diversidad biológica y a la fertilidad del suelo se asimila con los planteamientos conservacionistas de los grupos ecologistas, así como con la política restrictiva y punitiva que durante muchos años, ha desarrollado la administración medioambiental en esta zona. De este modo, la visión negativa que se tiene en la zona de la política de conservación de Espacios Naturales se ha trasladado a este método productivo (Lozano y Aguilar 2008).

"Hay quien dice aquí que habría que cambiarle el nombre y que entonces se desarrollaría más. Lo ecológico tiene connotaciones... muchas veces negativas porque, $\mathrm{mmmm}$, sin querer se une con medio ambiente (Consejería) y su manera de funcionar, que es el de poner multas. O sea, la relación de medio ambiente con los ciudadanos es ponerle multas (...). Lo ecológico se une con lo otro y no, no tiene una connotación positiva" (representante de la administración, 17/10/2004).

Se ha observado, por parte de la "población local", una cierta desconfianza hacia la agricultura ecológica por considerarla como fuente de conflicto social. Esta imagen, que deriva del enfrentamiento que se produjo entre los agricultores ecológicos y convencionales de Génave a finales de los años 80, ha supuesto un lastre para el desarrollo de la agricultura ecológica en la zona, sobre todo en el sector cooperativista, por miedo a que este desencuentro pudiera reproducirse en otras localidades.

"¿Meternos en lo ecológico? Para que nos pase como en Génave, las familias enfrentadas y todo eso" (Presidenta asociación de mujeres, 07/03/2006).

Sin embargo, no todas las opiniones son negativas, pues hay muchas personas de la comarca (entre las cuales no suelen estar la mayoría de los agricultores convencionales) que reconocen los aspectos positivos que se derivan de la aplicación de esta actividad y que valoran las potencialidades que la misma tiene para el desarrollo socioeconómico de la comarca, para una protección integral del patrimonio natural de la zona y de cara a promover una imagen diferencial del territorio.

\subsection{La legitimación de la actividad por parte del sector ecológico}

Los agricultores ecológicos del territorio responden a estas críticas utilizando argumentos que, en líneas generales, vienen a ajustarse al nuevo paradigma de desarrollo rural así como a los criterios establecidos por Conway (1985) para establecer la sustentabilidad de los agroecosistemas: productividad, estabilidad, sustentabilidad e igualdad. Reconocen que durante el período de reconversión el rendimiento de los cultivos es menor, lo que repercute en su aspecto. Unos procesos que son consecuencia lógica del cambio de manejo, dado que la agricultura industrial está estimulada de manera artificial, mientras que en la producción ecológica hay un mayor respecto a los ritmos naturales. Señalan que ambas cuestiones se van superando con los años a medida que la planta se ajusta al nuevo sistema productivo, asemejándose bastante a los convencionales en rendimiento y estética. Para muchos, constituye una satisfacción que sus olivares no se diferencien demasiado de los industrializados, si no es por la presencia de las botellas para el control natural de plagas, las banderas para identificar sus explotaciones y/o la hierba. Tratan de mostrar que los olivares van cambiando, con los años, el aspecto 
amarillento, por un tono verde semejante al de los convencionales y que, por tanto, son igual de "bonitos" que los otros.

"El comentario que se escuchaba es: 'son ecológicas'. Claro, se veía. Cuando veían como estaban decían: 'sí, muchos beneficios, muchas cosas, pero yo mi finca no la meto en eso'. Es que ahora mismo, ya te digo, se están arreglando" (agricultora ecológica, sub-unidad Sierra de Génave, 23/02/2005).

Como se puede observar en el cuadro 2, los agricultores contraponen a la imagen de suciedad y desidia atribuida a sus fincas, un discurso centrado en torno al esfuerzo y trabajo que supone el manejo correcto de "la hierba" y el mantenimiento del equilibrio adecuado en sus agroecosistemas. Hacen hincapié en que, mientras que "las olivas" convencionales están muertas, secas y estériles, las suyas están llenas de vida, como atestigua la abundante presencia de flora y fauna. Frente a los criterios exclusivamente economicistas del modelo de agricultura intensivo, utilizan otro baremo en el que las externalidades ambientales positivas de esta actividad pasan a ser un activo, un beneficio no cuantificable, pero que queda integrado, en su lógica, como un elemento intrínseco de la producción ecológica.

"Yo tengo un vecino allí, parejo conmigo, que tiene las olivas exactamente iguales, no hay una brizna de hierba. Las olivas se ven bonicas pero muertas. Tú vas al mío y ves las olivas verdes, no están amarillas, y no están sucias" (agricultor ecológico, sub-unidad Sierra de Segura, 10/03/2006).

En lo que refiere a la rentabilidad de sus cultivos, varios de los informantes han constatado que la oscilación de la producción es menor. Ello propicia que la cantidad de aceitunas obtenida en cada campaña sea constante y, por tanto, que los resultados económicos se mantengan estables en el tiempo. Una pauta que se ajusta a las directrices de la Agroecología ya que, según Altieri (1999), las estrategias deben orientarse a la optimización del agroecosistema y a la adaptación a las perturbaciones que puedan producirse en el mismo (heladas, sequía, etc.), y no tanto a buscar el rendimiento a corto plazo a base de maximizar las cosechas.

Se puede constatar que, frente al modelo industrial centrado en la cantidad y en la productividad, los agricultores ecológicos del territorio privilegian la calidad de sus procesos y productos, e integran como beneficios las externalidades ambientales positivas que este método productivo genera. De la misma forma, e intrínsecamente unido a lo anterior, frente a la inmediatez del paradigma productivista, valoran los efectos de esta actividad a medio y largo plazo, tanto sobre el ecosistema, como sobre la salud de agricultores y consumidores. Ello ha dado lugar a una cierta visión compartida de la producción ecológica entre los integrantes del sector como actividad menos contaminante, que permite obtener alimentos libres de residuos y que es más respetuosa con el medio ambiente. Estos agricultores manifiestan también una gran satisfacción y orgullo por el hecho de ser "ecológicos" y se identifican con el papel de "guardianes del medio ambiente" atribuido, desde la Unión Europea a este modelo de agricultura multifuncional. Una pauta que contrasta con la escasa valoración que buena parte de la sociedad española y muchos productores convencionales tienen del trabajo en el campo y de la profesión agraria.

"El orgullo de saber que estoy contribuyendo a que el medio ambiente sea más sano. A mis hijos que les encanta coger espárragos ya han aprendido que en cualquier olivar no se puede coger espárragos. En el mío hasta la gente va a coger espárragos. No van a sus olivas, van a las mías a cogerlos. Eso a mí me llena no la cartera, pero sí el orgullo, como agricultor respetuoso" (agricultor ecológico, sub-unidad Sierra de Segura, 15/02/2005).

\subsection{Visiones internas de la agricultura ecológica o cómo ser un "buen agricultor ecológico"}

A pesar de la existencia de esta cierta identidad compartida entre los miembros del sector, dentro del mismo hay concepciones diferenciales acerca de lo que caracteriza a un "buen" o "mal" agricultor ecológico. Desde ambas sub-unidades, así como por parte de algunos técnicos de desarrollo local, se critica a los productores que no hacen las labores en el momento preciso, ya que tienden a perpetuar la imagen de la agricultura ecológica como abandono de actividad, lo que repercute en todo el sector y desalienta a los productores que se están planteando la reconversión. Señalan que es necesaria una 
cierta profesionalización del sector ecológico, para lo cual resulta indispensable hacer los manejos necesarios, en el momento preciso y que los cultivos generen un mínimo de rentabilidad. Sin embargo, atendiendo a la configuración interna del sector, podemos apreciar opiniones diferentes.

Los integrantes de la sub-unidad Sierra de Génave consideran que los miembros de la sub-unidad Sierra de Segura no son "buenos" agricultores ecológicos porque no realizan un manejo adecuado del cultivo, lo que explicaría que la mayor parte de estas explotaciones no sean económicamente rentable, así como al fracaso de muchas de las iniciativas que se han implementado en la zona. Recurren, para hablar de los integrantes del otro grupo, a algunos de los términos utilizados por la población local para aludir a los agricultores ecológicos. En este sentido, creen que son poco realistas, al subordinar los aspectos económicos a otros factores como es la obtención de un sistema equilibrado, e indican que las prácticas que aplican estos agricultores ecológicos y, sobre todo, los denominados "ecologistas", son las que han propiciado que se desarrolle una imagen negativa de la misma en la zona.

El mantenimiento de las cubiertas herbáceas y sus implicaciones en el agroecosistema es el aspecto que más diferencia a los agricultores ecológicos de ambos grupos, tanto en su forma de concebir esta actividad como de hablar sobre ella. En la sub-unidad Sierra de Génave se utilizan prácticamente los mismos términos que se ha recogido en el discurso de los agricultores ecológicos para referirse a la misma: "la hierba nos invade", "las olivas están sucias". Ello viene a poner en evidencia que, más allá de los argumentos utilizados por este colectivo para explicar por qué no desarrollan este manejo: escasez de agua, olivares en pendiente, etc., en la práctica existe una visión peyorativa de la misma.

"A mí me parecen muy bien esas ideas, otra cosa es que se pueda hacer. Yo tengo que pensar primero en que nuestras rentas no pueden ir hacia abajo, de la de ninguno de nuestros socios, por mucho sostenible o mucho (...) Hay una cosa que se llama dinero y a ver cómo casamos las cosas. Esto sí es una realidad y lo otro no te digo que sea malo, pero está fuera de la realidad" (agricultor ecológico, sub-unidad Sierra de Génave, 25/10/2004).

Para los componentes de la sub-unidad Sierra de Segura, y para algunos técnicos de la zona, el hecho de que los integrantes del otro grupo no mantengan la cubierta vegetal deslegitima su carácter como "ecológicos". Al eliminarla, no sólo acentúan el problema de la erosión, sino que además se ven obligados a tener que adquirir fertilizantes en el mercado para reponer los nutrientes que el árbol necesita, ya que éste no los recibe a través de un suelo equilibrado. Estos agricultores consideran que "la hierba" es uno de los elementos fundamentales de regulación del agroecosistema y, por tanto, debería ser una de las normas de aplicación obligatoria en agricultura ecológica.

Destacan que los agricultores de Génave, al priorizar los beneficios económicos sobre los efectos ambientales, se limitan a realizar una práctica de sustitución de insumos, ya que la fertilización y el tratamiento de plagas y enfermedades se realiza mediante la aplicación de productos autorizados en agricultura ecológica. También se suele criticar al otro grupo por su escasa participación en las actividades que se desarrollan en la zona en torno a la agricultura ecológica, lo que ha ahondado en la falta de conexión del sector y ha obstaculizado la implementación de acciones colectivas.

"De considerar el cultivo, no como un máquina que tú le metes una cosa y sale un producto, sino considerarlo como un sistema más complejo en el que hay que, digamos tocar muchos resortes para lograr un equilibrio. Eso digamos que es, básico (...), incluso me atrevo a decir que en Génave que es donde más agricultura ecológica hay no se tiene muy claro" (agente de desarrollo local, 22/11/2004).

Podemos cerrar este epígrafe resaltando que en el territorio conviven diferentes percepciones de la producción ecológica en función de la adscripción de los entrevistados a la lógica predominante de los diferentes estilos agrarios existentes en la zona y a la forma de entender las relaciones culturanaturaleza. Por un lado, tenemos una concepción negativa de la producción ecológica dado que los atributos de la misma son analizados a partir de la lógica del cientifismo, racionalismo y atomismo que domina en el modelo agrario productivista. Esta visión es la que está más extendida entre la población 
local del territorio y, sobre todo, entre los agricultores convencionales. Esta lógica se reproduce, en menor medida, entre los integrantes de la sub-unidad Sierra de Génave cuya concepción de la producción ecológica se aproxima a la de sustitución de insumos. Por otro lado, encontramos una percepción de esta actividad que se caracteriza por integrar la perspectiva sistémica, así como a las directrices del holismo, subjetivismo, contextualismo y pluralismo que orientan formas de pensamiento alternativas (Noorgard 1987) y que tiene una mayor presencia entre los integrantes de la sub-unidad Sierra de Segura.

\section{Conclusiones}

A través de la mirada de diferentes actores sociales de la Sierra de Segura, se han analizado las representaciones sociales que se ha generalizado en torno a la producción ecológica, y se han identificado las percepciones que coexisten en el territorio en torno a dicha actividad, sus elementos constituyentes y sus implicaciones.

En primer lugar se puede destacar que la imagen generalizada de esta actividad en el territorio se sustenta, en primer lugar, en la "supuesta" mala apariencia de los cultivos bajo producción ecológica, así como en la menor rentabilidad de los mismos. Se suele considerar que los precios más elevados que alcanzan los alimentos ecológicos en el mercado y las ayudas complementarias a las que pueden acogerse, no alcanzan a compensar el descenso de su rendimiento ni la mayor carga de trabajo que conlleva. Una percepción que deriva, asimismo, de la preponderancia de la lógica de la modernización y a sus criterios productivistas, y del hecho de que los agricultores convencionales del territorio están acostumbrados a desarrollar unas técnicas "cómodas" que no requieren una labor de asesoramiento, formación y observación previa de los elementos del ecosistema y de sus interacciones, ni un cambio en los manejos.

En segundo lugar, hay que resaltar la importancia que posee la dimensión socio-cultural a la hora de plantearse la reconversión a este sistema productivo. Tal y como se ha mostrado, la presión social que se desarrolla sobre aquellas personas que voluntariamente deciden "señalarse" y "diferenciarse" del resto juega un papel muy importante a la hora desalentar a aquellos miembros de la comunidad que, aunque interesados en la producción ecológica, no quieren ser considerados diferentes, "los otros", "Ios hippies", pasando a ser objeto de la burla y la chanza tan habitual en estos casos.

Sin embargo, los problemas que presenta la agricultura ecológica en la Sierra de Segura no recaen, exclusivamente, en factores externos, sino que hay que tener en cuenta que el mismo sector se encuentra desarticulado y polarizado en torno a dos grupos, con visiones diferenciales de la producción ecológica, lo que impide establecer unas mínimas acciones colectivas para generar una estrategia territorial común.

El análisis de estas percepciones resulta, por tanto, fundamental no sólo para entender determinados factores que bloquean el desarrollo de la producción ecológica en este territorio y en Andalucía, sino también para comprender las posiciones divergentes que coexisten dentro del sector y la variabilidad de estrategias y posicionamientos entre sus integrantes.

\section{Notas}

1. Este trabajo ha sido financiado con los siguientes proyectos: "La comida invisible. representaciones sociales de los sistemas alimentarios: causas, consecuencias y transformaciones" (CSO2010-22074C03-02). Ministerio de Ciencia e Innovación y Fondos FEDER; “Territorio, calidad e innovación: el diseño 
de una nueva ruralidad europea". I+D (SEJ2007-63537/SOCI). Ministerio de Educación y Ciencia, y Fondos FEDER. Grupo de Investigación "Territorio, Cultura y Desarrollo" (TECUDE). Plan Andaluz de Investigación de la Universidad de Sevilla (P.A.I. SEJ-418).

2. El término "producción ecológica" se incluyó en el Reglamento (CE) № 834/2007 sobre producción y etiquetado de los productos ecológicos para abarcar la agricultura, ganadería, acuicultura y silvicultura ecológica, así como las diferentes etapas de la producción de alimentos ecológicos. Como este término es reciente, y la actividad más desarrolla en la zona de estudio ha sido la agricultura ecológica, utilizaremos indistintamente ambos vocablos: "producción ecológica" y "agricultura ecológica".

3. La reivindicación del conocimiento campesino en la generación de iniciativas de desarrollo rural sustentable es uno de los elementos más relevantes de la Agroecología. Frente a la las directrices de la agronomía convencional, que sustenta toda su lógica en los valores de la ciencia, las corrientes integradas dentro de la primera definición de la producción ecológica, entre las que estaría la perspectiva agroecológica abogan por el diseño participativo de estrategias de desarrollo que sean definidas desde las propias características locales y a partir de acciones colectivas (Norgaard 1987, Iturra 1988 y Hecht 1999.)

4. El primer país en crear una regulación para la agricultura ecológica fue Francia, en 1980. Su ejemplo fue seguido por Dinamarca en 1987 y por España en 1991. El desarrollo de normativas estatales, así como el crecimiento de la demanda de los alimentos ecológicos en Europa impulsaron a la Comunidad Económica Europea a elaborar el Reglamento (CEE) № 2092/91 sobre producción agrícola ecológica y su indicación en los productos agrarios y alimenticios que supuso la creación de un marco legal comunitario que detallaba los requisitos que debía cumplir un producto agrícola o un alimento para poder llevar algún tipo de referencia al método de producción ecológico.

5. De los actores sociales entrevistados, 29 fueron mujeres y 64 hombres. Para agilizar la lectura del texto se recurrirá a la utilización del masculino a modo de genérico sin pretender con ello invisibilizar el papel de las mujeres en los procesos analizados.

6. La Denominación de Origen Sierra de Segura se obtuvo en 1979, aunque se ratificó oficialmente hasta 1993.

7. A pesar de que los datos fueron recogidos entre 2005 y 2007 , las sucesivas vueltas al campo nos han permitido corroborar que el panorama que aquí presentamos se mantiene a grandes rasgos en la zona. Para obtener una visión más amplia de cambios operados a lo largo de los años y conocer con más detalle los manejos implementados por los integrantes del sector así como de las estrategias de coordinación verticales y horizontales desarrolladas, consultar Lozano y Aguilar (2012).

8. Aunque pudiera pensarse que esta denominación está asociada a la presencia de neorrurales en la zona, lo cierto es que prácticamente la totalidad de productores ecológicos de la zona son oriundos del mismo o se desplazaron al mismo por vínculos familiares, por lo que no se les pueden atribuir a los mismos los rasgos asociados a los neorrurales (Rodríguez y Trabada 1991).

\section{Bibliografía}

Acuña Delgado, Ángel (y Darío Ranocchiari)

2012 "Pastoreo trashumante. Práctica ecológica y patrimonio cultura, un estudio de caso", Gazeta de Antropología, vol. 28 (2), art. 12.

http://www.gazeta-antropologia.es/?p=4114

Altieri, Miguel Ángel

1999 "The ecological role of biodiversity in agroecosystems", Agriculture, Ecosystems \& Environment, vol. 
Banks, Jo (y Terrry Marsden)

2001 "The nature of rural development: the organic potential", Journal of Environmental Policy and Planning, vol. 3, n 2: 103-121.

Castañón, Manuel

2001 "Aspectos normativos de la producción ecológica", en Comité Andaluz de Agricultura Ecológica (ed.), La práctica de la agricultura y ganadería ecológicas. Sevilla, CAAE: 467-472.

Clunies-Ross, Tracey

1990 "Organic food: swimming against the tide?", en Terry Marsden y Jo Little (eds.), Political, Social and Economic Perspectives on the International Food System. Aldershot, Avebury Press: 200-214.

Conway, Gordon R.

1985 "Agroecosystem Analisis", Agricultural Administration, n² 20: 31-55.

Daly, Herman

1991 "Criterios operativos para el desarrollo sostenible", Debats, nº 35-36: 38-41.

Darnhofer, Ika

2005 "Organic Farming and Rural Development: Some Evidence from Austria", Sociologia Ruralis, vol. $45, n^{\circ} 4: 308-323$.

Foster, George

1974. "La sociedad campesina y la imagen del bien limitado", en Leopold Bartolomé y Enrique Gorostiaga (eds.), Estudios sobre el campesinado latinoamericano: la perspectiva de la Antropología Social. Buenos Aires, Ed. Periferia: 57-90.

García Trujillo, Roberto

2001 "Aproximación a las potencialidades y obstáculos de la agricultura y ganadería ecológicas en Andalucía", en Comité Andaluz de Agricultura Ecológica (ed.), La práctica de la agricultura y ganadería ecológicas. Sevilla, CAAE: 217-240.

Guthman, Julie

1998 "Regulating Meaning, Appropriating Nature: The Codification of California Organic Agriculture", Antipode, vol. 30, $\mathrm{n}^{\circ}$ 2: 135-154.

Guzmán, Gloria (y otros)

2000 Introducción a la Agroecología como desarrollo rural sostenible. Madrid, Mundi-Prensa.

Hammersley, Martyn (y Paul Atkinson)

2005 Etnografía: métodos de investigación. Barcelona, Paidós.

Hecht, Susanna

1999 "La evolución del pensamiento agroecológico", en Miguel Ángel Altieri (ed.), Agroecología. Bases científicas para una agricultura sustentable. Montevideo, Editorial Nordan-Comunidad: 15-30.

International Federation of Organic Agriculture Movements (IFOAM)

1989 Basic Standards of organic agriculture. Resolution of the General Assembly of the Iternational Federation of Organic Agriculture Movements. Ouagadougou, Burkina Faso, January 6 ${ }^{\text {th }}, 1989$.

Iturra, Raúl

1988 Antropología Económica de la Galicia Rural. Santiago de Compostela, Xunta de Galicia.

Jociles, María Isabel

1999 "Las técnicas de investigación en antropologia. Mirada antropológia y proceso etnográfico", Gazeta 
de Antropología, $\mathrm{n}^{\circ}$ 15, art. 1.

http://www.gazeta-antropologia.es/?p=3433

Kinsella, Jim (y otros)

2000 "Pluriactivity as a Livelihood Strategy in Irish Farm Households and its Role in Rural Development", Sociologia Ruralis, vol. 40, n 4: 481-496.

Lash, Scott (y John Urry)

1987 The end of Organized Capitalism. Cambridge, Polity.

Lozano, Carmen

2010 Agricultura Ecológica y "Segura". Nuevas estrategias de desarrollo en el medio rural andaluz. Jaén, Instituto De Estudios Giennenses.

2010 "Natural, tradicional y de la tierra. La promoción de la calidad agroalimentaria en los nuevos espacios rurales andaluces", en Marta Soler y Carmen Guerrero (coords), Patrimonio cultural en la nueva ruralidad andaluza. Sevilla, Instituto Andaluz de Patrimonio Histórico: 34-47.

2011 El sabor de la naturaleza. Agricultura ecológica en Parques Naturales Andaluces. Sevilla, Fundación Blas Infante.<br/p>2008 "Nuevas estrategias para nuevos contextos. Agricultura Ecológica en Parques Naturales Andaluces", Revista Sociedades Rurales, Producción y Medio Ambiente, vol. 8, $\mathrm{n}^{0}$ 16: 103-126.

2012 "Territorialising organic production. Collective actions and public policies in Andalusia", Sviluppo Locale, no 37-38: 45-66.

Morgan, Kevin (y Jonathan Murdoch)

2000 "Organic vs. conventional agriculture: knowledge, power and innovation in the food chain", Geoforum, vol. 31, n 2: 159-173.

Naredo, José Manuel

1983 "La crisis del olivar como cultivo "biológico"tradicional", Agricultura y sociedad, n² 26: 167-288.

Norgaard, Richard

1987 "The epistemological basis of agroecology", en Miguel Ágel Altieri (ed.), Agroecology. The Scientific Basis of Alternative Agriculture. Boulder, Wetsview Press: 21-28.

Ojeda, Mariano

2003 "Transformación agroecológica del olivar, comarca Sierra de Segura (Jaén): caracterización y diseño de estrategias". Trabajo de Fin de Carrera, Escuela Politécnica Superior de Huesca, Universidad de Zaragoza.

Pajarón, Manuel

2001 "Cultivo ecológico de olivar. El caso de Génave", en Comité Andaluz de Agricultura Ecológica, (ed.), La práctica de agricultura y ganadería ecológicas. Sevilla, CAAE: 251-257.

Ploeg, Jan Douwe

2008 The New Peasantries. Struggles for Autonomy and Sustainability in an Era of Empire and Globalization. London, Earthscan.

Ploeg, Jan Douwe (y otros)

2000 "Rural Development: From Practices and Policies towards Theory", Sociologia Ruralis., vol. 40, $n^{\circ}$ 4: 391-408.

Rodríguez Eguizabal, Angel Blas (y Xosé Elías Trabada Crende) 1991 "De la ciudad al campo: el fenómeno social neorruralista en España", Política y Sociedad, nº 9: 7386. 
1997 El análisis de la realidad en la intervención social: métodos y técnicas de investigación. Madrid, CCS.

Santamaría, Beatriz

2009 "De parques y naturalezas. Enunciados, cimientos y dispositivos", Revista de Dialectología y Tradiciones Populares LXIV (1): 297-324.

Sevilla Guzmán, Eduardo

1991 "Hacia un desarrollo agroecológico desde el campesinado", Política y sociedad, n 9: 57-72.

Toledo, Víctor

1993 "La racionalidad ecológica de la producción campesina", en Eduardo Sevilla y Manuel González de Molina (eds.), Ecología, Campesinado e Historia. Madrid, La Piqueta: 197-218.

Zimmermann, Jean-Benoît

1998 "Nomadisme et ancrage territorial: Propositions méthodologiques pour l'analyse des relations firmes-territoires", Revue d'Économie Régionale et Urbaine (RERU), nº 2 : 211-230. 